\title{
Simulation of the 3D Garment Based on the Dynamic Particle System
}

\author{
Qingfu LI \\ Department of Elements, Beijing Institute of Fashion Technology, Beijing, China
}

\begin{abstract}
Fabric simulation refers follow a similar principle by using physical models or mathematical models instead of the actual system to do experiments and research. The purpose of this project is model based on the particle system, according to the basic characteristics of the fabric drape deformation, by constructing bending, shear and other inner factors as well as the inherent gravity and other external constraints, combined with the rigid body collision theory of classical mechanics, a particle system modeling algorithm is given first, and then establish and implement a fabric simulation model, fabric model collisions between objects in contact with the other are calculated, thereby dynamic drape simulation graphics are generated.
\end{abstract}

KEYWORD: 3D Garment; Particle System; Spring-Mass Modeling

\section{INTRODUCTION}

Garment simulation model is the basis of garment simulation technology research, there are a lot of methods currently used for setting up garment simulation model. These methods mainly can be divided into geometric methods[1-4], physical methods[5] and hybrid methods. The early research work mainly focused on the modeling method based on geometric features. Geometry algorithm can simulate the certain properties of the garment, but there are still shortcomings that indispensable, it cannot simulate the appearance of the garment under different environment. Wei is probably the person who used geometric approach to computer graphics visualization of garment earliest. In 1986 Weil had used cosine curve and straw geometric transformation to simulate the suspension cloth, garment hanging on some constraints, using catenary computing shape when the cloth was free hanging, according to the actual need, through to constantly subdivision the triangle that construct by catenary and constraint point produces garment wrinkles, better simulation results have been achieved. JerryWeil's success has attracted many scholars to carry out the work of garment simulation and laid a solid foundation for the breakthrough of garment simulation research in the future, however, this method can only simulate appearance of the draping garment, cannot reflect the essence of truth of the garment. Breen[6-8] proposed the particle model, dividing the entire garment with geographic into grids, looking the intersection as a particle, using the minimum energy principle and optimized the most speed descent method to find the minimum energy state of the garment, namely the ultimate equilibrium state, and to realistically simulate the drape effect of garment made from different materials. Compared with other physical model, particle model is the most closest one to the nature of the garment, the shortage is that the calculation is too complicated and cost a long time, the energy expression of interaction between particles can be determined through complex test. Modeling method based on hybrid features is the combination of geometric method and physical method, it is in the process of pattern formation or simulation, with the geometric method to simulate the general outline of garment first, then use physical method to correct the finer structure, thereby gaining fidelity and fast graphics.

\section{SPRING-MASS MODELING}

The mass spring model is composed of a quadrilateral particle grid. There are three types of spring in the grid structure, which are structure spring, shearing spring and bending spring as well. Structure spring is the spring that connects neighboring particles either in the grid rows or in the grid columns, which is employed to keep the natural quadrilateral state of the grid. However, flat power 
exerting on the fabric cannot be completely passed to the whole piece of fabric by the structure spring. Thus the shearing spring is essential for the springmass modeling. The shearing spring is the spring that connects neighboring particles in the diagonal of each quadrilateral, which is proposed to facilitate the transmission of surface force. Desired cloth surface can be acquired by the effect of shearing spring. As the extension of structure spring, the blending spring connects spacing particles either in the grid rows or in the grid columns, which can prevent it from bending along the axial, and further prevent the cloth from being torn planarly. The spring-mass model of garment is illustrared in Figure 1.

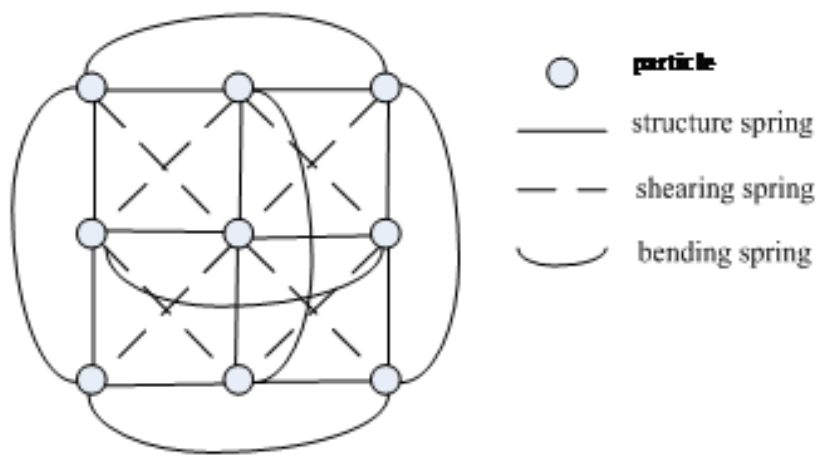

Figure1 Spring-mass model of garment

\subsection{Mechanical Analysis of Particles}

The resultant force $F(i, j)$ of particle $p(i, j)$ is the sum of internal force $F_{\text {in }}(i, j)$ and external force $F_{\text {out }}(i, j)$, which is formulated by

$$
F(i, j)=F_{\text {in }}(i, j)+F_{\text {out }}(i, j)
$$

Currently considered external forces in the cloth simulatuion are respectively gravity and air resistance. Each particle has the same quality, the quality is equal to the total quality of cloth divided by the total number of particle. In the process of cloth simulation, gravity roles in every particle is content. According to the law of resistance of stocke, air resistance fair is proportional to the $\mathrm{N}$ power of particle velocity. Internal force $F_{\text {in }}(i, j)$ of particle $p(i, j)$ includes spring force $F_{s}$ and damping force $F_{d}$. Particles in cells are connected by different springs, particles by each spring force and spring damping force are particles by internal force. If the free length between two endpoints of the spring is 1 , the modulus of elasticity is $\mathrm{k}$, then the elastic of endpoints in 3D space can be represented as follows:

$$
F_{s}=k\left[\left(s_{1}-s_{2}\right)-l \frac{s_{1}-s_{2}}{\left\|s_{1}-s_{2}\right\|}\right],
$$

where $s_{1}, s_{2}$ are the position vectors of endpoints.
For the internal particle $p(i, j)$ of spring-mass model, according to adding the interact with elastic of 12 related particles to calculate the particle by elastic as shown in Figure $1 . k_{1}, k_{2}, k_{3}$ are respectively the modulus of elasticity of structure spring, shear spring and bending spring. $l_{1}, l_{2}, l_{3}$ are respectively corresponding three types of freedom length of springs. The appropriate damping force is very important to maintain the stability of the system .For example, in order to prevent irregular vibration between two particles, a strong tension must be accompanied by a stable strong damping force. In the spring-particle model, damping force $F_{d}$ is proportional to the velocity difference of two connected particles.

\subsection{Numerical Solution of Dynamic Equation}

Assume that $m$ is the quality of underlying particle $p(i, j)$ at time $t$, the location of particle $p(i, j)$ is $s(i, j)$, the velocity of particle $p(i, j)$ is $v(i, j)$, the acceleration of particle $p(i, j)$ is $a(i, j)$, and the force acting on the particle $p(i, j)$ is $F(i, j)$. According to Newton's law, there is the relationship between acceleration and force of particle:

$$
a(i, j)=\frac{F(i, j)}{m} \text {. }
$$

Euler's method, median method and the fourth order runge-kutto method are classical methods to solve the numerical solution of dynamic equation according to the different accuracy. Via converting the continuous system to discrete structure based on spring-particle in spatial domain, Euler's method is used to solve the system equations in this paper. Based on the Euler method, there exists the following relationship between $\left.v(i, j)\right|_{t+\Delta t}$ at time instant $t+\Delta t$ and velocity $\left.v(i, j)\right|_{t}$ at time instant $t$ :

$$
\left.v(i, j)\right|_{t+\Delta t}=\left.v(i, j)\right|_{t}+\left.a(i, j)\right|_{t} \cdot t=\left.v(i, j)\right|_{t}+\frac{\left.F(i, j)\right|_{t}}{m} \cdot \Delta t
$$

The location of particle $p(i, j)$ at time instant $t+\Delta t$ is given by

$$
\left.s(i, j)\right|_{t+\Delta t}=\left.s(i, j)\right|_{t}+\left.v(i, j)\right|_{t+\Delta t} \cdot \Delta t
$$

Combining and solving equations (3), (4) and (5) yield

$$
\begin{gathered}
\left.a(i, j)\right|_{t+\Delta t}=\frac{\left.F(i, j)\right|_{t}}{m} \\
\left.v(i, j)\right|_{t+\Delta t}=\left.v(i, j)\right|_{t}+\left.a(i, j)\right|_{t} \cdot \Delta t \\
\left.s(i, j)\right|_{t+\Delta t}=\left.s(i, j)\right|_{t}+\left.v(i, j)\right|_{t+\Delta t} \cdot \Delta t
\end{gathered}
$$




\subsection{Collision Detection and Response}

In the process of fabric dynamic simulation, collision problem is not allowed to ignore. If there are no detection on collision in time, then the false phenomenon such as penetration and overlapping between objects will appear. Collision detection is also the important factors that affect the fabric simulation speed, the time it takes occupies about $40 \%$ of the total time typically. Thus the fit and unfit quality of collision problem directly related to the real-time and accuracy of the fabric simulation. This paper puts forward a kind of particle system that can generate new particles adaptively in any place where need to simulate the collision correctly. So compared with the general particle system, this model can obtain accurate simulation results only by needing a small amount of particles. At the same time, using coarse grid to do fast simulation becomes possible.

\section{SIMULATIUON EXPERIMENTS}

Given the quality of each particle, elastic coefficient of each spring and other parameters, the implementation steps of the cloth simulation experiments of the dynamic spring-particle model are as follows:

Step 1. Compute the acceleration of each particle.

Step 2. Compute the velocity of each particle.

Step 3. Compute the displacement of each particle.

Step 4. If all the particles reach equilibrium (the displacement less them a certain value), then stopping operation. Otherwise, continue step to perform step 3 .

The elastic coefficients for structure spring, shearing spring and bending spring are set to be 20 , 20 , and 20 respectively. The spring damping for structure spring, shearing spring and bending spring are set to be $0.2,0.2$, and 0.2 respectively. At the beginning of the simulation, the cloth was vertically placed, the upper left, right corner points are suspension point, then, hanging down slowly under the action of gravity. Figure 2 is the drape simulation results of garment.

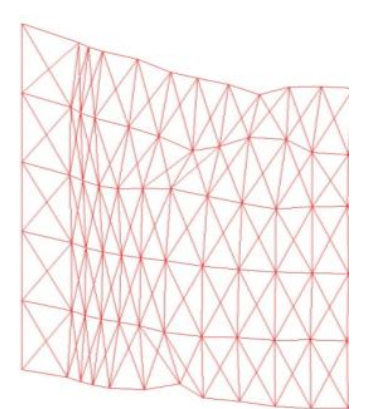

(a)Left

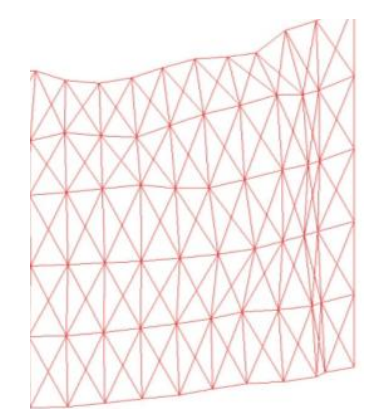

(b)right
Figure2 Drape simulation results of 3D garment

\section{CONCLUSIONS}

The basic approach is to establish an appropriate model of the mechanical structure of the fabric, then use mechanical theory derivation to show a threedimensional effect of the fabric and obtain relevant data, and then evaluate the performance of the fabric accordingly. To simulate nonlinear large deformation features of fabric by using cross-linked particle system simulation is a relatively recent emergence of a viable algorithm model. In this system, as long as reasonable construct constraints of energy or mechanical between the particles, a better differential equations of the particles motion will be able to get.

Three-dimensional fabric dynamic simulation technology enables the virtual world of the characters more real and natural, and it can effectively check the rational and fit of clothing styles, which is the premise of the three-dimensional garment computer aided design. Therefore, it has a very wide range of applications.

\section{ACKNOWLEDGEMENT}

This work is supported by the Scientific Research Key Program of Beijing Municipal Commission of Education under Grant No. KZ201210012014, the Project of Youth Fund of Scientific Research Innovation (No. 2014AL-32) and the Project of Beijing Institute of Fashion Technology for Mathematics, Physics and Mechanics Innovative Teaching Team.

\section{REFERENCES}

[1] J WELL. The synthesis of cloth objects. Computer Graphics (Proc. Siggraph), 1986, 20:49 -54.

[2] T AGUI, NAGAO Y, NAKAJMA M. An expression method of cylindrical cloth objects. An expression of folds of a sleeve using computer graphics. Trans Soc of Electronics, Information and Communications, 1990, J73D- П (7): 1095-1097.

[3] NG N H, GRIMSDALE R L.Computer graphics techniques for modelling cloth. IEEE Computer Graphics and Applications, 1996, 16:36.

[4] HINDS B K, McCARTNEY J. Interactive garment design. Visual Computer, 1990, 6:53 -61.

[5] FEYNMAN C.Modeling the appearance of cloth. Cambridge: Cambridge Mass, 1996.

[6] BREEN D E. A particle_based model for simulating the draping behavior of woven cloth. Rensselaer Polytechnic Inst, 1993.

[7] D BARAFF, W ITKIN A. Large step s in cloth s imu lat ion. Proceedings of SIGGRAPH, 1998. 43- 54. 